\title{
A Novel Routing Algorithm for Wireless Sensor Network Using Particle Swarm Optimization.
}

\author{
Snehal Sarangi ${ }^{1}$, Biju Thankchan ${ }^{2}$ \\ ${ }^{12}$ Department Of Computer Science and Engineering \\ ${ }^{12}$ Disha Institute Of Management and Technology,Raipur
}

\begin{abstract}
Wireless sensor network is becoming a progressively Important and challenging research area. Advancement in WSN enable a wide range of environmental monitoring and object tracking system. Wireless sensor networks consists of small low cost sensor nodes, having a limited transmission range and their processing, storage capabilities and energy resources are limited. We consider energy constrained wireless sensor network deployed over a region. The main task of such a network is to gather information from node and transmit it to base station for further processing. Generally, it needs a fixed amount of energy to receive one bit of information and an additional amount of energy to transmit the same. This additional amount depends on the transmission range. So, if all nodes transmit directly to the $B S$, then they will quickly deplete their energy. To perform routing in wireless sensor network with this limitation of low power, energy and storage capabilities is a major problem. Many solutions has been proposed where energy awareness is essential consideration for routing. The LEACH, PEGASIS, GROUP, Ant colony optimization etc has provided elegant solutions and has shown very effective results. In this paper, we have proposed a Particle Swarm Optimization based Routing protocol (PSOR) where we have taken energy efficiency as major criteria for performing routing and deriving optimized path for data forwarding and processing to base node. The PSOR generates a whole new path of routing by taking energy as fitness value to judge different path and choose best optimized path whose energy consumption is less as compared to other routing paths. We concluded with the result obtained by performing experiment on our proposed algorithm PSOR and comparing its result with Genetic Algorithm which shows better result as compared to Genetic Algorithm and the experiments performed are done using NS2 simulator.
\end{abstract} Keywords: Particle Swarm Optimization, Routing, Optimization, Genetic Algorithm, wireless sensor network.

\section{Introduction}

Recent advances in sensing, computing and communication technologies coupled with the need to continuously monitor physical phenomena have led to the development of Wireless Sensor Networks (WSNs). WSN consist of four main components: A radio, a processor, sensors and battery. A WSN is formed by densely deployed sensor nodes in an application area. In most deployments, the sensor nodes have self-organizing capabilities, to form an appropriate structure in order to collaboratively perform a particular task. Wireless Sensor Networks (WSNs) is a class of wireless ad hoc networks in which sensor nodes collect, process, and communicate data acquired from the physical environment to an external Base Station (BS)..The key constraints in the development of WSNs are limited battery power, cost, memory limitation, limited computational capability, and the physical size of the sensor nodes. Due to the severe energy constraints of large number of densely deployed sensor nodes, it requires a suite of network protocols to implement various network control and management functions such as synchronization, node localization, and network security. Wireless Sensor Networks are found suitable for applications such as surveillance, precision agriculture, smart homes, automation, vehicular traffic management, habitat monitoring, and disaster detection. Routing in wireless sensor networks differs from conventional routing in fixed networks in various ways: There is no infrastructure, wireless links are unreliable, sensor nodes may fail, and routing protocols have to meet strict energy saving requirements.

The traditional routing protocols have several shortcomings when applied to WSNs, which are mainly due to the energy-constrained nature of such networks. In this paper, we attempt to overcome limitations of the wireless sensor networks such as: limited energy resources, varying energy consumption based on location, high cost of transmission, and limited processing capabilities which forms the basic draw backs while sending or receiving data packets during routing. Besides maximizing the lifetime of the sensor nodes, it is preferable to distribute the energy dissipated throughout the wireless sensor network in order to minimize maintenance and maximize overall system performance while routing. In wireless sensor networks each node do not save a fixed energy, even the shortest path of the network takes a huge energy which leads to the permanent failure and reduce the lifetime of the network. So energy efficiency and distance should be consider as major criteria for optimized path selection. 


\section{Literature Survey}

The optimization of network parameters for a WSN routing process to provide maximum network lifetime might be considered as a combinatorial optimization problem. Many researchers have recently studied the collective behavior of biological species such as ants as an analogy providing a natural model for combinatorial optimization problems. Selcuk Okdem et al[1] developed routing using ant colony optimization (ACO) . Ant colony optimization (ACO) algorithms simulating the behavior of ant colony have been successfully applied in many optimization problems such as the asymmetric traveling salesman, vehicle routing , and WSN routing. Singh et al. [2] proposed an ant based algorithm for WSN routings. However, this algorithm does not consider the main specifics of WSN structures, including energy related issues. Zhang et al. [3]proposed ant based algorithms for WSNs; their study includes three routing algorithms named SC, FF, and FP. The algorithms are successful with initial pheromone settings to have a good system start-up, but the SC and FF algorithms are not quite effective in latency, while providing better energy efficiency. Besides, the FP algorithm, while providing high success rates of data delivery, consumes much higher energy than the FC and FF algorithms. The Energy Efficient Ant Based Routing Algorithm for WSNs (EEABR) [4], based on a ACO meta heuristic, is another proposed ant based algorithm to maximize the lifetime of WSNs. The algorithm uses a good strategy considering energy levels of the nodes and the lengths of the routed paths. Heinzelman et al [5] developed a cluster based routing scheme called Low Energy adaptive cluster in hierarchy In LEACH the role of the cluster head is periodically transferred among the nodes in the network in order to distribute the energy consumption. The performance of LEACH is based on rounds. Then, a cluster head is elected in each round. For this election, the number of nodes that have not been cluster heads and the percentage of cluster heads are used. Once the cluster head is defined in the setup phase, it establishes a TDMA schedule for the transmissions in its cluster This scheduling allows nodes to switch off their interfaces when they are not going to be employed. The cluster head is the router to the sink and it is also responsible for the data aggregation. As the cluster head controls the sensors located in a close area, the data aggregation performed by this leader permits to remove redundancy. A centralized version of this protocol is LEACH-C [6]. This scheme is also based on time rounds which are divided into the set-up phase and the steady-phase. In the set-up phase, sensors inform the base station about their positions and about their energy level. With this information, the base station decides the structure of clusters and their corresponding cluster heads. Since the base station posses a complete knowledge of the status of the network, the cluster structure resulting from LEACH-C is considered an optimization of the results of LEACH Lindsey et al [7] developed a Power-Efficient Gathering in Sensor Information Systems (PEGASIS) . It is considered an optimization of the LEACH algorithm. Rather than classifying nodes in clusters, the algorithm forms chains of the sensor nodes. Based on this structure, each node transmits to and receives from only one closest node of its neighbors. With this purpose, the nodes adjust the power of their transmissions The node performs data aggregation and forwards it the node in the chain that communicates with the sink. In each round, one node in the chain is elected to communicate with the sink. The chain is constructed with a greedy algorithm. Ayan Chakraborty et al [8] proposed a genetic algorithm inspired routing protocol(GROUP) which shows enhanced performance in terms of energy efficiency and network life time over another schemes.

In this paper, we proposed a particle swarm optimization based routing(PSOR).PSOR algorithm is designed to maximize the lifetime of WSNs. The algorithm uses a good strategy considering energy levels of the nodes and the lengths of the routed paths. In this paper, we have compared the performance results of our PSOR approach to the results of the Genetic algorithm. Various differently sized networks are considered, and our approach gives better results than Genetic algorithm(GROUP)[ 8] in terms of energy consumption. The main goal of our study was to maintain network life time at a maximum, while discovering the shortest paths from the source nodes to the base node using a particle swarm based optimization technique called PSO, Furthermore instead of forming greedy chain or cluster of chain, which may not always ensure minimum energy dissipation, we make use of intelligent particle swarm based optimization Particle swam optimization is an inspiration from the flocking birds. Here also we have multiple search agents that try to search for the global minima in the fitness landscape a shukla et al [9], just like the GA[10].The result obtained by PSOR shows encouraging improvement over Genetic Algorithm[GROUP], PEGASIS ,LEACH and ACO.The PSOR algorithm was implemented in network simulator 2 for ubuntu platform.

The rest of the paper is organized as follows: section 2 PSO, section 3 Proposed Routing Algorithm using PSO, Section 4Simulation Section 5 Experimental result and enhancement, Section 6 Conclusion and Future work..

\section{Particle Swarm Optimization}

The particle swarm optimization algorithm, originally introduced in terms of social and cognitive behavior by Kennedy and Eberhart (1995) [11], solves problems in many fields, especially engineering and computer science. The individuals, called particles henceforth, are flown through the multidimensional search space with each particle representing a possible solution to the multi-dimensional optimization problem. Each solution's fitness is based on a performance function related to the optimization problem being 
solved. The movement of the particles is influenced by two factors using information from iteration-toiteration as well as particle-to-particle. As a result of iteration-to- iteration information, the particle stores in its memory the best solution visited so far, called pbest, and experiences an attraction towards this solution as it traverses through the solution search space. As a result of the particle-to particle interaction, the particle stores in its memory the best solution visited by any particle, and experiences an attraction towards this solution, called gbest, as well. The first and second factors are called cognitive and social components, respectively .After iteration, the pbest and gbest are updated for each particle if a better or more dominating solution (in terms of fitness) is found. This process continues, iteratively, until either the desired result is converged upon, or it is determined that an acceptable solution cannot be found within computational limits. For an $\mathrm{n}$ dimensional search space, the ith particle of the swarm is represented by an $\mathrm{n}$-dimensional vector, $X i=(x i 1, x i 2 \ldots . . . x i n)$.

The velocity of this particle is represented by another $\mathrm{n}$ dimensional vector $V i=(v i 1 ; v i 2 \ldots . . . v i n) T$. The previously best visited position of the ith particle is denoted as $P i=(p i l, p i 2, \ldots \ldots . . p i n) T$. ' $g$ ' is the index of the best particle in the swarm. The velocity of the ith particle is updated using the velocity update equation given by

$\mathbf{v}_{\text {id }}=\mathbf{v}_{\mathrm{id}}+\mathbf{c}_{1} \mathbf{r}_{1}\left(\mathbf{p}_{\mathrm{id}}-\mathbf{x}_{\mathrm{id}}\right)+\mathbf{c}_{2} \mathbf{r}_{2}\left(\mathbf{p}_{\mathrm{gd}}-\mathbf{x}_{\mathrm{id}}\right)$

and the position is updated using

$\mathbf{x}_{\mathrm{id}}=\mathbf{x}_{\mathrm{id}}+\mathbf{v}_{\mathrm{id}}$

where $d=1,2 \ldots . . n ; i=1 ; 2 \ldots . S$, where $S$ is the size of the swarm; $c 1$ and $c 2$ are constants, called cognitive and social scaling parameters respectively (usually, $c 1=c 2 ; r 1, r 2$ are random numbers, uniformly distributed in $[0,1])$. Equations (2.1) and (2.2) are the initial version of PSO algorithm. A constant, Vmax, is used to arbitrarily limit the velocities of the particles and improve the resolution of the search. Further, the concept of an inertia weight was developed to better control exploration and exploitation. The motivation was to be able to eliminate the need for Vmax. The inclusion of an inertia weight $(w)$ in the particle swarm optimization algorithm was first reported in the literature in 1998 (Shi and Eberhart, 1998) [13]. The resulting velocity update equation becomes:

$v_{i d}=w * v i d+c 1$ r1 $($ pid $-x i d)+c 2 r 2(p g d-x i d)$

Eberhart and Shi (2000) [12] indicate that the optimal strategy is to initially set $w$ to 0.9 and reduce it linearly to 0.4 , allowing initial exploration followed by acceleration toward an improved global optimum.

\section{Proposed Routing Algorithm Using Pso :}

Particle swarm optimization is mainly a computational method that optimizes a problem by iteratively trying to improve a candidate solution with regard to given measure of quality. For solving any optimization problem we have to first formulate the problem according to optimization problem. In this proposed algorithm we have to choose the best path according to fitness value which is according to the minimum distance to be travelled by a data up to base node, since we are dealing with energy efficient routing ,more the distance more the energy will be lost in sending data. So to calculate fitness value we are using PSO and generating an optimum path taking consideration in all sensor nodes.

\section{A) FITNESS FUNCTION}

To find optimize path using PSO, we need to find the fitness value of each path .This fitness value will be used to select the local best and global best for PSO. The path having minimum fitness value will be the best optimal solution.

Fitness value $=\operatorname{dist}(i, j)+\operatorname{dist}(j$, base $)$

where $\mathrm{i}, \mathrm{j}$ are the node distance.

\section{B) REPRESENTATION}

As the PSO equation given in above equation 2.1 and equation 2.2 works on real number values ,but for generating path it is easier to use natural number system so we are using Shortest positioning index to make node position very simple, In shortest position indexing the values are sorted from minimum value to maximum value and position is given accordingly.

For example

$\left[\begin{array}{lllll}0.923 & 0.422 & 8.32 & 3.66 & 2.55\end{array}\right]$

Applying shortest positioning index

$\left[\begin{array}{lllll}2 & 1 & 5 & 4 & 3\end{array}\right]$


C)ALGORITHM :

\section{PARTICLE SWARM OPTIMIZATION BASED ROUTING.}

Phase $1:$ [ Initialization Phase]

for ( $\mathrm{s}=0$ to number of solutions or populations).

for ( $\mathrm{d}=0$ to number of sensor nodes).

Randomly solutions are selected.

Compute new route using solution.

End for .

Compute fitness value of initialized solution.

Compute global best and Local best.

End for .

Phase 2 :[Update Phase]

while criteria does not match

for ( $\mathrm{s}=1$ to number of solutions)

for ( $d=1$ to number of sensor nodes).

update solution using PSO update equation.

Generate new path based on update solution.

End for.

Compute fitness value for updated route.

Compute global best and local best.

End for.

Note the global best

End while.

To find best optimal path with least energy usage we have used Particle Swarm Optimization (PSO).We set an initial solution by selecting a random number of solutions from the set of $\mathrm{x}$ ! solutions ; $\mathrm{x}$ is the total number of solutions. After getting initial random solutions we calculate fitness value of each solution, according to equation (3.2.1).After that we calculate best among the entire solution and set it as initial global and local best.PSO update equation is used to update old solution and generate new solutions and their nodes are calculated. These solutions along with their nodes are then used to find the fitness value of each solution. The process will be repeated till the given iteration is satisfied. Based on this continuous iteration and fitness value the solution which is better is replaced than its other solutions.

\section{Simulation}

The scenario was simulated using network simulator (NS2)[13] based on network topology.25 wireless sensor nodes were deployed onto $50 \times 50 \mathrm{~m}^{2}$ grid as shown in fig 1.The Particle swarm optimization routing algorithm is implemented under NS2.Program is written in OTcl programming language.

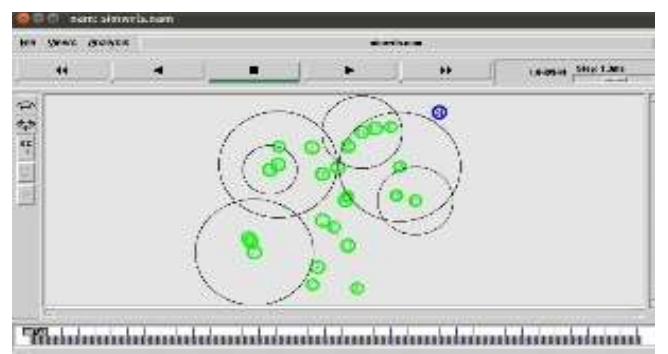

Fig 1 Graphical Representation of network topology.

\section{Experimental Result And Discussion}

This section focuses on the efficiency of the proposed algorithm PSOR with the Genetic Algorithm[10]. This section shows the experimental results and the parameters setting of the proposed algorithm.

\section{A) EXPERIMENTAL SETUP}

For every algorithm there are some control parameters which are used for efficient working. The first control parameter is maximum number of population or solutions which we have taken its value to be 30 .The next parameter in our experiment is number of sensor nodes which we have taken as 30 nodes. Another control parameter is number of iteration, which we have taken in our experiment is 3000 .The fourth control parameter is number of random solution to be chosen, which in this experiment we have taken 30.The next control parameter is the value of c1 and c2 which we have taken as $\mathbf{2}$ for both. And inertia weight w is also a 
A Novel Routing Algorithm for Wireless Sensor Network Using Particle Swarm Optimization.

parameter and we have taken its value as 0.7. The comparison of the results are with the genetic algorithm result where in the experiment the crossover probability of GA taken is 0.7 .We have keep on changing the parameter of number of nodes, number of random solutions and iterations, to keep on observing the fitness value and compare the results with the fitness value of GA.

\section{B) EXPERIMENTAL RESULTS.}

Table 1 Fitness value calculated by PSOR and GA for varying number of nodes.

\begin{tabular}{|l|l|l|l|l|}
\hline NODES & 10 & 15 & 20 & 25 \\
\hline GA & 1989.59 & 4190.62 & 5136.21 & 6807.21 \\
\hline PSOR & 1425.41 & 3083.45 & 3815.16 & 5093.67 \\
\hline
\end{tabular}

Here we are provided with different number of nodes and comparing it with fitness value of PSOR with GA.

Table 2 Fitness value calculated by PSOR and GA for varying number of iterations.

\begin{tabular}{|l|l|l|l|l|}
\hline iteration & 1000 & 1500 & 2500 & 3000 \\
\hline GA & 5832.24 & 5606.07 & 6533.35 & 6533.35 \\
\hline PSOR & 4731.42 & 4823.93 & 4280.07 & 4280.07 \\
\hline
\end{tabular}

Table 3 Fitness value calculated by PSOR and GA for varying number ofrandom solution

\begin{tabular}{|l|l|l|l|l|}
\hline solution & 10 & 15 & 20 & 25 \\
\hline GA & 5694.36 & 6641.24 & 6376.04 & 6432.24 \\
\hline PSOR & 4720.25 & 5007.34 & 4655.08 & 4497.21 \\
\hline
\end{tabular}

\section{C) DISCUSSION}

From table 1,2,3 it is concluded that proposed algorithm PSOR gives better result than Genetic Algorithm with varying number of nodes, iterations and random solutions. Parameters written above is standard parameter we have measured from experiment.

\section{Conclusion And Future Enhancement:}

In this paper we proposed a novel scheme for sensor networks which results in energy efficient routing across the network. The concept of this model is based on the fact is greater the distance travelled to send data more is the consumption of sensor energy. The algorithm is done by using concept of PSO . Our results prove that after a considerable optimum path can be calculated using PSO which shows better result than GA giving us best routing path with least distance to be travelled. As future work this PSO can be hybrid with existing algorithm ,it may perform better than the existing.

\section{References}

[1] Selcuk Okdem and Dervis Karaboga : Routing in Wireless Sensor Networks Using an Ant Colony Optimization (ACO) Router Chip:In Sensors 2009.

[2] Shio Kumar Singh, M P Singh, and D K Singh : Energy Efficient Homogenous Clustering Algorithm for Wireless Sensor Networks: International Journal of Wireless \& Mobile Networks ( IJWMN ), Vol.2, No.3, August 2010.

[3] Y. Zhang, L. D. Kuhn, and M. P. J. Fromherz, "Improvements on Ant Routing for Sensor Networks," M. Dorigo et al. (Eds.): ANTS 2004, Springer-Verlag Berlin Heidelberg 2004, vol. LNCS 3172, pp. 154-165, 2004.

[4] Tiago Camilo, Carlos Carreto, Jorge Sá Silva, Fernando Boavida : An Energy-Efficient Ant-Based Routing Algorithm for Wireless Sensor Networks.

[5] Rabiner, W.; Kulik, J.; Balakrishnan, H. Adaptive Protocols for Information Dissemination in Wireless Sensor Networks. In Proceedings of the Fifth Annual International Conference on Mobile Computing and Networking (MOBICOM), Seattle, WA, USA, August, 1999; pp. 174-185.

[6] Heinzelman, W.B.; Chandrakasan, A.P.; Balakrishnan, H. An Application-Specific Protocol Architecture for Wireless Microsensor Networks. IEEE Trans. Wirel. Commun. 2002, 1, 660-670.

[7] Lindsey, S.; Raghavendra, C.S. PEGASIS: Power-Efficient Gathering in Sensor Information Systems. In Proceedings of the Aerospace Conference, Big Sky, MT, March, 2002; pp. 1125-1130.

[8] Ayon Chakraborthy,Swarup kumar Mitra,Mrinal Kanti Niskar:A Genetic Algorithm Inspired routingProtocol for Wireless sensor Network: in International Journal of Computational IntelligenceTheory and practice,Vol 6No.1 June 2011.

[9] A. Shukla et al.: Towards Hybrid and Adaptive Computing, SCI 307, pp. 187-207. springerlink.com @ Springer-Verlag Berlin Heidelberg 2010

[10] Manoj Kumar1, Mohammad Husian2, Naveen Upreti3 \& Deepti Gupta :GENETIC ALGORITHM: REVIEW AND APPLICATION:In International Journal of Information Technology and Knowledge Management July-December 2010, Volume 2, No. 2, pp. 451-454

[11] Kennedy, J., Eberhart, R.: Particle swarm optimization. In:Neural Networks,1995. Proceedings., IEEE International Conference on. vol. 4, pp. 1942\{1948. IEEE(1995)

[12] Shi, Y., Eberhart, R.: A modi_ed particle swarm optimizer. In: Evolutionary Computation Proceedings, 1998. IEEE World Congress on Computational Intelligence.The 1998 IEEE International Conference on. pp. 69\{73. IEEE

[13] S. Mccanne, S. Floyd, and K. Fall, "Network Simulator 2 (NS-2) version 2.28," in http://www-nrg.ee.lbl.gov/ns/, http://www.isi.edu/nsnam/ns (1998) 